\title{
KAMUTHE video microanalysis system for use in Brazil: translation, cross-cultural adaptation and evidence of validity and reliability
}

\begin{abstract}
BACKGROUND
KAMUTHE is a video microanalysis system which observes preverbal communication within the music therapy setting. This system is indicated for children with autism spectrum disorder (ASD) or multiple disabilities. The purpose of this study was to translate, adapt to Brazilian Portuguese language and analyze some psychometric properties (reliability and validity evidence) of KAMUTHE administration in Brazil for individuals with ASD.
\end{abstract}

\section{PARTICIPANTS AND PROCEDURE}

Translation, back translation, analysis by judges, and pilot application were performed to obtain evidence of content and face validity. The second part of this study was to administer KAMUTHE in 39 consecutive children with ASD. An individual session of improvisational music therapy was applied to assess the different behaviors included in KAMUTHE. The intra-rater reliability, concurrent validity and convergent validity were analyzed.

\section{RESULTS}

Translation and cross-cultural adaptation were followed and some cultural adaptations were needed. Inter-rater re-

\begin{abstract}
liability was very good (ICCs 0.95-0.99) for the three child's behaviors analyzed. Criteria validity with a moderate negative association was found $(r=-.38, p=.017)$ comparing the behavior "Gazes at therapist" and the level of ASD along with the Childhood Autism Rating Scale (CARS). Convergent validity was established between the behavior "Gazes at therapist" and the two nonlinguistic communication scales (social interaction and interests) of the Children's Communication Checklist (CCC) with a moderate correlation $(r=-.43, p=.005)$.
\end{abstract}

CONCLUSIONS

The administration of the KAMUTHE video microanalysis system showed positive results in children with ASD. Further studies are needed to improve the reliability and validity of the instrument in Brazil.

KEY WORDS

music therapy; communication; KAMUTHE; video microanalysis; autism spectrum disorders

ORganization - 1: Universidade do Estado de Santa Catarina, Florianópolis City, Brazil · 2: Universidade Federal do Rio

Grande do Sul, Porto Alegre, Brazil

AUthors' CONTRIBUtions - A: Study design - B: Data collection - C: Statistical analysis - D: Data interpretation .

E: Manuscript preparation · F: Literature search · G: Funds collection

Corresponding Author - Gustavo Schulz Gattino, Ph.D., Universidade do Estado de Santa Catarina, 2007 Avenida Madre Benvenuta, 8803500 Florianópolis City, Brazil, e-mail: mt.gattino@gmail.com 


\section{BACKGROUND}

Children with autism spectrum disorder (ASD) differ in terms of level and characteristics of preverbal communication (DiStefano \& Kasari, 2016; Woynaroski et al., 2016). Abnormalities in some brain structures (e.g. basal ganglia and mirror neuron system - MNS) (Chugani, 2012), number of treatments, the impact of family and peer relationships and cognitive impairments could directly affect the functioning of preverbal communication in this population (Travers et al., 2012). Common impairments in this area among children with ASD include difficulties in expression and comprehension of gestures, facial expressions and vocalizations (Boucher, 2012; Farmer, 2016). These children usually exhibit some kind of vocal sounds (Prelock \& Nelson, 2012; Stewart et al., 2016). However, these sounds could not present an intention to communicate (Grossi, Marcone, Cinquegrana, \& Gallucci, 2012). One example of this case is echolalia, where there is speech repetition of information heard externally (Grossi et al., 2012). Children with ASD display deficits to infer emotional states (anger, sadness and joy, for example) from facial expressions (Brewer et al., 2016; Carter, Hyde, Williams, \& Hodgins, 2016). In relation to gestures, these children might exhibit pacing, hand-flapping or clapping as well as head shaking (Aldaqre, Schuwerk, Daum, Sodian, \& Paulus, 2016; Dimitrova, Özçalışkan, \& Adamson, 2016). A core nonverbal deficit in children with ASD is limited joint attention behaviors (Mundy, Kim, McIntyre, Lerro, \& Jarrold, 2016; Murza, Schwartz, Hahs-Vaughn, \& Nye, 2016). Joint attention involves coordinated and mutual attention between two people toward an object or event through eye gaze, pointing or showing (Kasari, Gulsrud, Freeman, Paparella, \& Hellemann, 2012). Children with low functioning ASD could ignore the presence of objects and/or events in some cases, which decreased the possibilities of interaction (Boucher, 2012).

Music interaction as a nonverbal means of communication may build a framework to assess children with ASD (Bergmann \& Burghardt-Distl, 2016; Caria, Venuti, \& de Falco, 2011). These children display particular characteristics in auditory functioning with normal processing of the primary auditory cortex (related to music) and a decrease in the secondary auditory cortex (related to speech) (Kujala, Lepistö, \& Näätänen, 2013; Ludlow et al., 2014; Samson et al., 2011). In this sense, music might facilitate communication skills providing a comprehensive means to engage (Wigram, 2010; Wigram \& Gold, 2006). Moreover, there is evidence to suggest that many individuals with ASD show a preference for music and are able to understand simple and complex emotions (Allen, Davis, \& Hill, 2013; Molnar-Szakacs \& Heaton, 2012). In other words, there is a dissociation between emotional recognition abilities in musical and social domains in children with ASD (Molnar-Szakacs \& Heaton, 2012). As an example, the MNS, which exhibits abnormalities in individuals with autism and is involved in emotional recognition, might be engaged in the musical experiences, because these experiences activate brain regions that overlap with the MNS (Koelsch, 2009; Wan, Demaine, Zipse, Norton, \& Schlaug, 2010). In summary, the music assessments allow one to verify some nonverbal skills which would not be possible without music making in children with ASD, because the particular auditory processing (more connected with music) and the emotional recognition linked to music experiences create different possibilities to engage in comparison with the traditional assessments (Wigram, 2002; Wigram \& Lawrence, 2005). The Cochrane review published by Geretsegger, Elefant, Mössler, and Gold (2014) provided evidence that music therapy may help children with ASD to improve their skills in areas that constitute the core of the condition including social interaction, verbal communication, initiating behavior, and social-emotional reciprocity. Music therapy may also help to enhance non-verbal communication skills within the therapy context. In the last 17 years music therapy (MT) has presented evidence of the use of music to assess nonverbal communication according to different tools and by three explicit music-based diagnostic instruments: Wigram's Harper House Music Therapy Assessment (Wigram, 2000), Music Therapy Diagnostic Assessment (MTDA) (Oldfield, 2006) and Music Based Scale for Autism Diagnostics (MUSAD) (Bergmann et al., 2015). Among those assessment tools (including the explicit music-based diagnostic instruments), only a few of them have emerged with acceptable psychometric properties (Mauat, Gattino, \& Riesgo, 2014). The MUSAD presented the best psychometric properties, showing a complex analysis of test-retest reliability, and confirmatory factor analysis (Bergmann et al., 2015).

\section{KAMUTHE ASSESSMENT}

KAMUTHE (KAtegoriensystem MUsikTHErapie) is a video microanalysis system which observes preverbal communication within the MT setting (Plahl, 2004). This system is indicated for children with ASD or multiple disabilities (Plahl, 2007). This measurement tool displays a very detailed coding system to assess the client's and music therapist's preverbal behaviors. The communicative behaviors of the child are categorized into gaze, musical activity, vocalizations and gestures (Table 1). The behavior of the music therapist is categorized into musical, verbal and nonverbal communicative behavior. Tables 1 and 2 describe the music therapist's and child's behaviors, respectively. In addition, the microanalysis 
Table 1

Communication categories of the music therapist

\begin{tabular}{lcc}
\hline Musical behavior & Verbal behavior & Nonverbal behavior \\
\hline MUS $>1$ & VER $>1$ & NON $>1$ \\
Vocalizing & Verbal comment & Gesturing \\
MUS $>2$ & VER $>2$ & NON $>2$ \\
Playing on instruments & Praising the child & Offering an instrument \\
MUS $>3$ & VER $>3$ & NON $>3$ \\
Singing a song & Asking the child & Moving an instrument \\
MUS $>4$ & VER $>4$ & NON $>4$ \\
Singing a song and & Inviting the child & Moving the child \\
accompanying on an instrument & &
\end{tabular}

Table 2

Communication categories of the child

\begin{tabular}{|c|c|c|c|}
\hline Gaze & Play/musical activity & Vocalizations & Gestures \\
\hline $\begin{array}{l}\mathrm{BLI}>1 \\
\text { Gazes at instrument }\end{array}$ & $\begin{array}{c}\text { SPI }>1 \\
\text { Touching an instrument }\end{array}$ & $\begin{array}{c}\text { VOK > } 1 \\
\text { Vocalizing }\end{array}$ & $\begin{array}{c}\text { GES }>1 \\
\text { Conventional } \\
\text { gesture }\end{array}$ \\
\hline $\begin{array}{l}\text { BLI }>2 \\
\text { Gazes at therapist's face }\end{array}$ & $\begin{array}{c}\text { SPI }>2 \\
\text { Creating sound with } \\
\text { an instrument }\end{array}$ & $\begin{array}{l}\text { VOK > } 2 \\
\text { Singing }\end{array}$ & $\begin{array}{c}\text { GES }>2 \\
\text { Unconventional } \\
\text { gesture }\end{array}$ \\
\hline $\mathrm{BLI}>3$ & $\mathrm{SPI}>3$ & VOK $>3$ & \\
\hline Gazes at an object & Playing with objects & Talking & \\
\hline $\begin{array}{l}\mathrm{BLI}>4 \\
\text { Gazes at therapist }\end{array}$ & $\begin{array}{c}\text { SPI }>4 \\
\text { Moving instruments } \\
\text { on arms (e.g. bells) }\end{array}$ & $\begin{array}{l}\text { VOK > } 4 \\
\text { Laughing }\end{array}$ & \\
\hline $\begin{array}{l}\text { BLI > } 5 \\
\text { Gazes at mother/father }\end{array}$ & $\begin{array}{c}\mathrm{SPI}>5 \\
\text { Moving rhythmically }\end{array}$ & $\begin{array}{l}\text { VOK > } 5 \\
\text { Moaning }\end{array}$ & \\
\hline $\begin{array}{l}\mathrm{BLI}>6 \\
\text { Gaze around the room }\end{array}$ & $\begin{array}{c}\text { SPI }>6 \\
\text { Guided by therapist } \\
\text { in moving }\end{array}$ & $\begin{array}{l}\text { VOK > } 6 \\
\text { Crying }\end{array}$ & \\
\hline
\end{tabular}

rating is based on frequency and duration of different behaviors.

The KAMUTHE application is divided into the following steps (Plahl, 2007): 1) Defining and selecting the sample of sequences within the MT sessions (parts of the session that will be analyzed), 2) Choosing the program of analysis and technique of coding (software or method used), 3) Training application of category system and assessing reliability (inter-rater) and 4) Analyzing different parameters at a micro level (frames, seconds and minutes). The theoretical framework for the construction of the category system stemmed from the communicative modalities of the concept of music in Orff Music Therapy (Plahl, 2007). These modalities are related to the ancient concept of musiké - comprising word, sound and movement as verbal, musical and nonverbal modal- ities. Thus, all musical activities have been defined as communicative activities comprising each kind of sound expression through voice or instrument or movement expression. In constructing the category system for MT, KAMUTHE, it took into account the findings of Towle, Farran and Comfort (1988), who gave considerations on observational coding systems in the interaction between parents and children with multiple disabilities (Towle, Farran, \& Comfort, 1998). Relating to psychological concepts of interaction and transaction, formal criteria in constructing the category system were: 1) possible interaction units and their possible functions in the MT process and 2) patterns of reference and regulation in MT and their dimensions in time.

According to the statements above, the purpose of this study was to translate, adapt to Brazilian
KAMUTHE video microanalysis system for use in Brazil 
Portuguese language and analyze some psychometric properties (reliability and validity evidence) of KAMUTHE administration in Brazil for individuals with ASD.

\section{PARTICIPANTS AND PROCEDURE}

\section{KAMUTHE TRANSLATION \\ AND CROSS-CULTURAL ADAPTATION}

Gustavo Schulz Gattino,

Alexandre Mauat da Silva,

Felipe Grahl

Figueiredo,

Lavinia SchülerFaccini
The different methods of translation and cross-cultural adaptation (Guillemin, Bombardier, \& Beaton, 1993; Sperber, 2004; Wild et al., 2005) differ along with the general characteristics of the instrument (e.g., type of instrument, fulfillment by the client or therapist), level of complexity (which may include simple or complex analysis in terms of content validity evidence), the presence of an external committee to analyze the different translation procedures and the professional responsible for carrying out the translation (a specialist in translation or an expert in the field related to the measurement who is fluent in different languages). The KAMUTHE version in Brazilian Portuguese was developed combining different models of translation, using the operative (with minimum stages included in the translation process) method proposed by Sperber (2004) and the proposal established by Guillemim et al. (1993) and Wild et al. (2005), who postulate the necessity of judges' analysis.

The translation was carried out in line with the following steps: 1) permission from the author of the original assessment; 2 ) the production of two translators' versions independently, which were then compared by the researchers who compiled a composite version (version 1); 3) back translation into the original language by an English native speaker fluent in Portuguese and who had not participated in the previous stages and was not in contact with the original text; 4) review of the back translation, harmonization with the previous versions and development of version 2 ; 5) review by the expert committee in terms of clarity and relevance of each behavior to obtain the face validity (a source of content validity evidence) and development of version 3; 6) reconciliation and development of the final version. The cross-cultural adaptation was established during the whole review process and in the development of all different versions (a sources of content validity evidence).

\section{SAMPLE}

Children meeting the following inclusion criteria were recruited for the study: 1) confirmed diagnosis of ASD according to DSM-IV-TR and 2) aged between 4.0 and 14.0 years (APA, 2002). The exclusion criteria were intolerance to sounds or music and profound hearing loss. The ASD diagnoses were assigned by a multidisciplinary team consensus conference according to the DSM-IV diagnostic research criteria for autism, Asperger syndrome or pervasive developmental disorder - not otherwise specified (APA, 2002). The multi-disciplinary team consisted of at least one psychiatrist and one neurologist who was experienced in the field of ASD. Diagnosis was based on all available data, including medical histories, psychiatric and physical examinations, information of the Brazilian Childhood Autism Ratting Scale, CARSBR (Pereira, Riesgo, \& Wagner, 2008) and in some cases, data of the Autism Diagnostic Interview Revised (Becker et al., 2012). With the intention to find a Spearman correlation of at least $r=.40$ between the category "Gaze at therapist (BLI > 4)" of KAMUTHE and the total score of the Brazilian Childhood $\mathrm{Au}$ tism Rating Scale (CARS-BR), we estimated a sample size of 39 participants for an alpha error of .05 and power of .80 . The justification for that estimated sample size is based on the number of participants described in two validation studies in Brazil, specific for individuals with ASD. The study of Pereira et al. (2008) presented a sample of 60 participants and that of Becker et al. (2012) had 20 participants.

\section{PROCEDURE}

Data collection with KAMUTHE was conducted at three different special schools that specialized in educational care for children with ASD in Porto Alegre, Brazil. In addition to basic education, these specials schools offer assessment and treatment for children with ASD in speech therapy and occupational therapy. Diagnostic classification of ASD was conducted in accordance with the diagnostic research criteria for mental disorders proposed by DSM-IV (APA, 2002) in combination with scores of the Brazilian Childhood Autism Rating Scale, CARS-BR (Pereira et al., 2008). The KAMUTHE assessment took place in three different rooms (one in each special school). These rooms were big and equipped with a predefined set of standard MT instruments arranged according to the KAMUTHE intervention protocol manual (Gattino, 2012). The KAMUTHE system created by Plahl (2007) did not have an intervention protocol. However, we believe that is necessary to standardize the application of a measurement tool in order to facilitate the application of KAMUTHE by different music therapists and allow the comparison between different individuals.

This manual was organized in six activities and based on improvisational music therapy (IMT). The intervention protocol is composed of one session of 30 minutes which is based on a session model of the Creative Music Therapy Approach (Nordoff-Robbins) (Aigen, 2009). In this sense, the session starts with 
a small conversation with the child and is followed by the hello song. At the next 3 moments, the therapist follows the child's musical-emotional lead, interacting with a guitar, with the objective to create music and with musical instruments (one drum, one guitar and one piano). The session ends with a goodbye song and with another small conversation to ask the child how the session was. The KAMUTHE manual is part of a published dissertation (Gattino, 2012) and is included as an appendix to this paper. The KAMUTHE sessions were carried out by three music therapists who were previous trained in the intervention protocol.

All sessions were videotaped to allow better observation of nonverbal behaviors and scoring of KAMUTHE as well as to gain material to assess interrater reliability. All observations were carried out by two raters and all scorings were carried out after all sessions. One rater analyzed 19 sessions while the other observed 20 sessions. KAMUTHE raters analyzed the minutes 5, 10, 15, 20 and 25 of all sessions (full five minutes). The inter-rater reliability was determined to establish a similar understanding of the KAMUTHE scoring with the analysis of the same 7 sessions independently by the two different assessors. Within the 12 behaviors for the music therapist and 20 for the child described in KAMUTHE, there were selected 3 behaviors addressed to children and to music therapists. The reason for this choice is because the inclusion of all behaviors would require a very long analysis that is not the intention of this research, whose main goal is to present the first validity evidence of KAMUTHE in Brazil. The raters analyzed frequency and duration of different behaviors in each period of five minutes observed. The behaviors of the music therapist were playing on instruments (MUS > 2) and singing a song and accompanying on an instrument (MUS $>4$ ). The child's behaviors were singing (VOK $>2$ ), creating sound with an instrument (SPI $>2$ ), and gazing at therapist $(\mathrm{BLI}>4)$. The reason for choosing these behaviors is due to the clear possibility to detect and discern from other behaviors included in the KAMUTHE system. Moreover, the analysis did not include more behaviors in order to increase the quality of the observations made by the two independent raters.

\section{OTHER MEASURES}

To assess the validity evidence of KAMUTHE the subjects were examined with the following two tools: the Brazilian Childhood Autism Rating Scale (CARSBR) and the Children's Communication Checklist revised (CCC). The Brazilian Childhood Autism Rating Scale (CARS-BR) (Pereira et al., 2008) is a 15-item rating scale that aids in the identification of children with autism and that distinguishes them from other children with compromised development but without autism. The scale assesses behaviors in 14 domains usually affected in autism and a single category for general impressions of autism. These 15 items are as follows: relating to people, imitation, emotional response, body use, object use, adaptation to change, visual response, listening response, taste, smell, and touch response and use, fear or nervousness, verbal communication, nonverbal communication, activity level, level and consistency of intellectual response and, finally, general impressions. The scores assigned to each domain vary from 1 (within the limits of normality) to 4 (severe autistic symptoms). The total score varies from 15 to 60 and the cutoff point for autism is 30. CARS-BR was conducted in an interview with one of the parents of the child with ASD and was carried out by one research assessor. The Children's Communication Checklist revised (CCC) (Malerbi, 2008) is a 70-item checklist that assesses children's communication behaviors (children had to be speaking in multiword phrases) across nine subscales: speech, syntax, inappropriate initiation, coherence, stereotyped conversation, use of context, rapport, social relationships and interests. The last two subscales were called nonlinguistic scales (social interaction and interests) and reflected autistic-type behaviors that might justify further investigation. Each item was a statement of behavior and respondents were asked to judge whether this: definitely applied (2 points), applied somewhat (1) or did not apply (0). An 'unable to judge' option is also available for each item. A score of 132 or below is used as a cutoff point for pragmatic language impairment. The Brazilian translation and validation study of CCC found a mean score of 55.87 in typical children for nonlinguistic scales (social interaction and interests). In this sense, it is expected that individuals with ASD present the lowest score.

\section{DATA ANALYSIS}

According to the standards for educational and psychological testing established by the American Educational Research Association (AERA), American Psychological Association (APA) and National Council on Measurement in Education (NCME) published in 2014 , a comparative analysis was conducted to verify two primary sources of validity evidence within the MT observation assessment instrument: 1) test content, 2) relation to other variables and 3) internal structure (AERA, APA, \& NCME, 2014). Validity evidence based on test content refers to traditional forms of content validity evidence, such as the rating of test specifications and test items. In this research we carried out the cross-cultural adaptations (conducted during the translation process) and the face validity as different sources of test content validly
KAMUTHE video microanalysis system for use in Brazil 
Gustavo Schulz Gattino,

Alexandre Mauat da Silva,

Felipe Grahl Figueiredo, Lavinia SchülerFaccini
(Conti, Scagliusi, Queiroz, Hearst, \& Cordás, 2010). Relation to the variables is a validation process that refers to traditional forms of criterion-related validity evidence, such as concurrent validity. In addition, a comprehensive investigation of the relationships among test scores and other variables, such as convergent validity, was conducted. Internal structure refers to a statistical analysis of item and score subdomains to investigate the primary and secondary (if any) dimensions measured by an assessment. Internal structure evidence could evaluate the "strength" or "salience" of the major dimensions underlying a measurement tool by the use of statistics to evaluate precision such as test reliability, decision accuracy and consistency, generalizability coefficients, conditional and unconditional standard errors of measurement, and test information functions (Carpente, Manne, \& Gattino, 2016).

Validity evidence based on test content. This source of evidence was established by cross-cultural adaptation (described in the KAMUTHE translation) and by face validity which was carried out by an external committee of two experts who observed the clarity and relevance of each behavior described in KAMUTHE (Gattino, 2012). Clarity was assessed by a 5-point Likert scale with the following scoring: 1 (I did not understand anything), 2 (I just understood a small part), 3 (I understood a part), 4 (I understood almost everything), and 5 (I understood perfectly and I have no doubt). Moreover, the relevance was descried by a 4-point Likert scale with the ratings: 1 (not relevant), 2 (quite relevant), 3 (relevant) and 4 (really relevant).

Validity evidence based on relations to other variables. The second part of the validity process included concurrent validity. Along with the standards for educational and psychological testing (AERA, APA,

Table 3

Sample demographic and clinical characteristics

\begin{tabular}{lc}
\hline Characteristic & $n(\%)$ \\
\hline Gender & \\
$\quad$ Male & $28(72)$ \\
Female & $11(28)$ \\
$\begin{array}{l}\text { Age (years) } \\
\text { (mean, } \pm \text { standard deviation) }\end{array}$ & $11.54( \pm 3.12)$ \\
Education & \\
$\quad \begin{array}{l}\text { Special school } \\
\quad \text { Does not attend school }\end{array}$ & $38.00(97.40)$ \\
$\begin{array}{l}\text { General score of CARS-BR } \\
\text { (mean, } \pm \text { standard deviation) }\end{array}$ & $37.32( \pm 4.61)$ \\
$\begin{array}{l}\text { Nonlinguistic CCC scales } \\
\text { (mean, } \pm \text { standard deviation) }\end{array}$ & $53.53( \pm 3.80)$ \\
\hline
\end{tabular}

\& NCME, 2014), the concurrent validity deals with the degree to which an assessment is correlated with a previously validated measure. This source of validity evidence allows for comparisons of different measures that represent different but related constructs and evaluates measures that are taken at the same time. Concurrent validity was established comparing the total scores of CARS-BR with the three children's behaviors analyzed in five minutes (singing, creating sound with an instrument and gazing at the therapist) in frequency and duration according to the Spearman correlation. All comparisons of behaviors included from the KAMUTHE system were established in line with frequency and duration (in seconds) of each behavior.

The next part of the analysis was examining convergent validity. The standards for educational and psychological testing (AERA, APA, \& NCME, 2014) postulate that convergent validity occurs when two measures are significantly correlated with each other, positively or negatively, thus showing evidence of similarity or identity of the evaluated constructs. Therefore, the scales of the all children's behaviors in five minutes (singing, creating sound with an instrument and gazing at the therapist) and music therapist's behavior in five minutes (playing on instruments and singing a song and accompanying on an instrument) were compared with each other through the Spearman correlation. In addition, the children's behaviors in five minutes were compared with the CCC nonlinguistic scales (social interaction and interests).

Validity evidence based on internal structure. This study also assessed inter-rater reliability where two raters analyzed 7 sessions of KAMUTHE assessment ( $18 \%$ of all observations) in the three behaviors examined in the child according to the intraclass correlation coefficient (ICC) (Kim, Wigram, \& Gold, 2009). The music therapist's behaviors were not analyzed due their explicit observation.

This research was part of a larger study on the effects of MT on nonverbal communication of children with ASD (Figueiredo, 2014; Gattino, 2012) which was approved by the local ethics committee (at Porto Alegre Clinical Hospital, Porto Alegre, Brazil) with the number 09280 and was conducted according to the recommendations of the Declaration of Helsinki (Diniz \& Corrêa, 2001).

\section{RESULTS}

Table 3 presents the sample demographic and clinical characteristics of the 39 participants with ASD.

Face validity. In the clarity assessment, it was expected that $75 \%$ of items observed by experts would present a score between 4 and 5 (Conti et al., 2010). For relevance, it was expected that $75 \%$ of the as- 
sessed items would present scores between 3 and 4 (Conti et al., 2010). If these data were not achieved, the final review process needed to be revised. The analysis of experts obtained the expected scores in clarity $(75 \%)$ and relevance (83\%). Minor modifications were carried in the KAMUTHE instrument after the analysis of judges.

Concurrent validity. According to the different analysis, there was found one statistically significant result with a moderate negative association $(r=-.38, p=.017)$ comparing the behavior duration of "gazes at the therapist" and the level of autism along with the Childhood Autism Rating Scale (CARS). In other words, the general level of autism assessed by the CARS-RS is lower when the child presents more manifestations of gazes at the therapist. The other comparisons of concurrent validity were not statistically significant.

Convergent validity. The association between the child's and music therapist's behaviors (in frequency and duration) only showed two statistically significant results. The result was observed between the behaviors "creating sound with an instrument" (SPI > 2) and "singing a song and accompanying on an instrument" (MUS > 4) with a correlation for duration of $r=-.48(p<.001)$ and for frequency of $r=-.40$ $(p<.001)$. This means that the more the music therapist plays and sings, the less the child participates in playing together. For the comparisons of the child's behaviors with the CCC nonlinguistic scales there was found only one statistically significant result, which was established between the behavior duration of the "gazes at therapist" and the two nonlinguistic communication scales (social interaction and interests) of the Children's Communication Checklist (CCC) with a moderate correlation $(r=-.43$, $p=.005)$. This result indicates that when the child displays more behaviors of "gaze at the therapist" his/ her score in social interaction and interests of CCC decreases (lower scores in these two scales represent a decrease of autistic communication characteristics).

Inter-rater reliability. All behaviors exhibited very good results according to the intraclass correlation coefficient (ICC) for frequency and duration. Table 4 presents the results of inter-rater reliability.

\section{DISCUSSION}

The KAMUTHE assessment presented in this article is a good proposal, in terms of psychometric properties, for an instrument to allow the comprehension and measurement of the nonverbal communication construct. The final version of the instrument presented a good face validity which allows very good intraclass correlation coefficients for inter-rater reliability. This evidence of reliability is in consonance with the study of Kim et al. (2009), which evaluated
Table 4

Inter-rater reliability

\begin{tabular}{lc}
\hline Child's behavior & Agreement ICC \\
\hline $\begin{array}{l}\text { Creating sound with an } \\
\text { instrument }(\mathrm{SPI}>2)\end{array}$ & $0.99 / 0.99$ \\
frequency/duration & \\
$\begin{array}{l}\text { Gazes at therapist }(\mathrm{BLI}>4) \\
\text { frequency/duration }\end{array}$ & $0.95 / 0.95$ \\
$\begin{array}{l}\text { Singing }(\mathrm{VOK}>2) \\
\text { frequency/duration }\end{array}$ & $0.99 / 0.99$ \\
\hline
\end{tabular}

emotional, motivational and interpersonal responsiveness of children with autism using a microanalysis method (ICC of 0.59-0.98). Moreover, these results might be explained by the use of an intervention protocol manual that standardized the different moments of MT sessions, facilitating the observation of the different raters (Wosch \& Wigram, 2007). In relation to the concurrent validity, the study found a moderate negative association between the general score of CARS-BR and the behavior "gaze at therapist". The finding is in accordance with the existent literature on communication for individuals with ASD where distinct publications postulate that gaze contact is a central ability to detect autistic symptoms and associated behaviors (d'Arc et al., 2016; Pell et al., 2016; Thorup, Nyström, Gredebäck, Bölte, \& Falck-Ytter, 2016). Two validated instruments in MT (Individual Music-Centered Assessment Profile for Neurodevelopmental Disorders and Music-based Autism Diagnostics) for children with ASD consider gaze contact as a core characteristic to be detected by musical interaction (Bergmann et al., 2015; Carpente, 2014). The two other behaviors analyzed, singing and creating a sound with an instrument, are important for the music interaction, but perhaps are not essential to detect diagnostic traits in children with ASD due to the absence of significance in comparison with the CARS-BR general scores. Even though KAMUTHE uses a microanalysis perspective, it is not possible to claim that KAMUTHE has sensitivity to be used as a diagnostic tool. Futures studies (using other MT assessments together with KAMUTHE) need to be performed to confirm this hypothesis. In accordance with the convergent validity between the behaviors of the music therapist and child, only two results showed statistical significance. This results indicate a typical situation of an MT assessment session (Wigram \& Gold, 2006). It is tricky to define in a quantitative perspective the difference between the musical behaviors from the therapist in relation to the child's interaction (Wosch \& Wigram, 2007), and even more if the posited situation is the first session of the MT assessment (that was the case presented in this research). For this reason, Plahl (2007) suggests
KAMUTHE video microanalysis system for use in Brazil 
Gustavo Schulz Gattino,

Alexandre Mauat da Silva,

Felipe Grahl Figueiredo, Lavinia SchülerFaccini that the relation between music therapist and the child need to be assessed in a qualitative approach, which is in line with three principles, resonance, synchronicity and reciprocity, that indicate the level of interaction in the musical play. This analysis was not carried out in this research, because the intention in this first application of KAMUTHE in Brazil was to observe all behaviors quantitatively (Kim, Wigram, \& Gold, 2008; Kim et al., 2009). The moderate negative association found in the comparison of creating sound with an instrument (SPI > 2) and singing a song and accompanying on an instrument (MUS > 4) for frequency and duration point to the difficulty of the music therapist to engage the child with ASD in the musical play at the beginning of the MT process. Therefore, KAMUTHE would help to detect the first objectives of the MT assessment related to the musical play. Wigram, Pedersen and Bonde (2002) suggest that one of the uses of the MT assessment is precisely to detect goals to be explored in the next MT sessions. The moderate correlation $(r=-.43, p=.005)$ established between "gazes at therapist" frequency and the two nonlinguistic communication scales (social interaction and interests) of CCC highlights again the capacity of KAMUTHE to identify gaze deficits in children with ASD through musical play, because the same behavior presented a significant correlation for the concurrent validity with the CARS-BR. The absence of significance found in other correlations with the CCC may suggest that musical behaviors of KAMUTHE could not be connected with preverbal autistic traits. Further investigations are necessary to verify whether KAMUTHE has sensitivity to relate its musical behaviors assessed with the core preverbal characteristics of autism. It is important to clarify that one measurement tool does not need to have complete sensitivity and specificity to detect traits in one domain (Gattino, Walter, \& Schüler-Faccini, 2010). For this reason, one assessment tool could be used in combination with another one. Only the MUSAD assessment (which is specific for individuals with ASD) has evidence comparing ASD screening scales with MT tools used in MT assessments. In line with KAMUTHE results, the MUSAD study did not find strong correlations between the domains assessed, comparing ASD scales and this MT measurement tool. Two different validity studies are investigating at this moment the use of MT assessments (Individual Music-Centered Assessment Profile for Neurodevelopmental Disorders, IMCAP-ND and Individualized Music Therapy Assessment Profile, IMTAP) to detect nonverbal communication in children with ASD and will establish the convergent validity with different scales for ASD screening (Carpente et al., 2016; Mauat et al., 2014). It is expected that the results of these studies will help to show the potential of KAMUTHE assessment among the different sources of validity from other MT tools. The current study was the first official investigation of validity of KAMUTHE worldwide. Previous studies conducted by Plahl $(2004,2007)$ indicated inter-rater statistics, but did not carry out measures with the intention to validate this instrument. Unlike the original study, this investigation created an intervention protocol to apply the instrument with the intention to standardize its application. Additional studies will be essential to verify whether this protocol is necessary and whether it works in different cultures, since it was created in Brazil.

\section{LIMITATIONS OF THE STUDY}

This investigation did not include a qualitative analysis for the music therapist and child's behaviors during the musical play. For future research a mixed analysis is suggested that considers qualitative analysis to observe the resonance, synchronicity and reciprocity (levels of interactions established by Plahl (2007) to interpret the results of the KAMUTHE analysis) with an objective analysis described by frequency and duration, that were used in previous clinical trial studies of microanalysis in MT. Moreover, this study did not include all child's and music therapist's behaviors of KAMUTHE. Inclusion of this could point out additional directions that may start other research hypotheses of KAMUTHE. The next studies need to consider a shorter age range to observe whether there are specificities of KAMUTHE according to a close age range in comparison with other scales.

\section{CONCLUSIONS}

The administration of the KAMUTHE video microanalysis system showed positive results in individuals with ASD in Brazil as stated by its official Brazilian Portuguese translation. The evidence of inter-rater reliability, content, and concurrent and convergent validity of this tool exhibited consistent psychometric properties which allow one to use KAMUTHE in the MT clinical practice and research field. Further studies are needed to improve the reliability and validity of the instrument in Brazil.

\section{ACKNOWLEDGMENTS}

This work was supported by FIPE/HCPA (project no. 09280) and by the Brazilian Research Council (CNPq).

\section{References}

Aigen, K. (2009). Verticality and containment in song and improvisation: an application of schema the- 
ory to Nordoff-Robbins music therapy. Journal of Music Therapy, 46, 238-267. doi: 0022-2917-46-3-238

Aldaqre, I., Schuwerk, T., Daum, M. M., Sodian, B., \& Paulus, M. (2016). Sensitivity to communicative and non-communicative gestures in adolescents and adults with autism spectrum disorder: saccadic and pupillary responses. Experimental Brain Research, 234, 1-13.

Allen, R., Davis, R., \& Hill, E. (2013). The effects of autism and alexithymia on physiological and verbal responsiveness to music. Journal of Autism and Developmental Disorders, 43, 432-444.

American Educational Research Association, American Psychological Association, \& National Council on Measurement in Education. (2014). Standards for educational and psychological testing. Washington, DC: American Educational Research Association.

American Psychiatric Association. (2002). Manual diagnóstico e estatístico de transtornos mentais: texto revisado (DSM-IV-TR) [Diagnostic and Statistical Manual of Mental Disorders]. Artmed.

Becker, M. M., Wagner, M. B., Bosa, C. A., Schmidt, C., Longo, D., Papaleo, C., \& Riesgo, R. S. (2012). Translation and validation of autism diagnostic interview-revised (ADI-R) for autism diagnosis in Brazil. Arquivos de Neuro-Psiquiatria, 70, 185-190.

Bergmann, T., \& Burghardt-Distl, A. (2016). Music in diagnostics: using musical-interactional settings for diagnosing autism. Nordic Journal of Music Therapy, 25 (Suppl.), 98-99.

Bergmann, T., Sappok, T., Diefenbacher, A., Dames, S., Heinrich, M., Ziegler, M., \& Dziobek, I. (2015). Music-based autism diagnostics (MUSAD) - a newly developed diagnostic measure for adults with intellectual developmental disabilities suspected of autism. Research in Developmental Disabilities, 43, 123-135.

Boucher, J. (2012). Research review: structural language in autistic spectrum disorder - characteristics and causes. Journal of Child Psychology and Psychiatry, 53, 219-233. doi: 10.1111/j.14697610.2011.02508.x

Brewer, R., Biotti, F., Catmur, C., Press, C., Happé, F., Cook, R., \& Bird, G. (2016). Can neurotypical individuals read autistic facial expressions? Atypical production of emotional facial expressions in autism spectrum disorders. Autism Research, 9, 262-271.

Caria, A., Venuti, P., \& de Falco, S. (2011). Functional and dysfunctional brain circuits underlying emotional processing of music in autism spectrum disorders Cerebral Cortex, 21, 2838-2849.

Carpente, J. A. (2014). Individual music-centered assessment profile for neurodevelopmental disorders (IMCAP-ND): new developments in music-centered evaluation. Music Therapy Perspectives, 32, 56-60. doi: 10.1093/mtp/miu005
Carpente, J., Manne, S., \& Gattino, G. (2016). Validity and reliability evidence for a music therapy observational instrument for children with autism spectrum disorder. New York: American Music Therapy Association.

Carter, E. J., Hyde, J., Williams, D. L., \& Hodgins, J. K. (2016). Investigating the influence of avatar facial characteristics on the social behaviors of children with autism. In J. Kaye (ed.), Proceedings of the 2016 CHI Conference on Human Factors in Computing Systems (pp. 140-151). New York, NY, USA: ACM.

Chugani, D. C. (2012). Neuroimaging and neurochemistry of autism. Neuroimaging and Neurochemistry of Autism, 59, 63-73.

Conti, M. A., Scagliusi, F., Queiroz, G. K., Hearst, N., \& Cordás, T. A. (2010). Adaptação transcultural: tradução e validação de conteúdo para o idioma português do modelo da Tripartite Infl uence Scale de insatisfação corporal [Cross-cultural adaptation: translation and Portuguese language content validation of the Tripartite Influence Scale for body dissatisfaction]. Cadernos de Saúde Pública do Rio de Janeiro, 26, 503-513.

Dimitrova, N., Özçalışkan, Ş., \& Adamson, L. B. (2016). Parents' translations of child gesture facilitate word learning in children with autism, Down syndrome and typical development. Journal of $A u-$ tism and Developmental Disorders, 46, 221-231.

Diniz, D., \& Corrêa, M. (2001). The Helsinki Declaration: relativism and vulnerability. Cadernos de Saúde Pública, 17, 679-688.

DiStefano, C., \& Kasari, C. (2016). The window to language is still open: distinguishing between preverbal and minimally verbal children with ASD. Perspectives of the ASHA Special Interest Groups, 1, 4-11.

Farmer, K. J. (2016). Effect of music vs. nonmusic paired with gestures on spontaneous verbal and nonverbal communication skills of children with autism between the ages 1-5 (dissertation's thesis). Florida State University, Florida, USA.

Figueiredo, F. G. (2014). Musicoterapia improvisacional aplicada à comunicação pré-verbal de crianças com transtornos do espectro autista: ensaio controlado e randomizado (master's thesis) [Improvisation Music Therapy on preverbal communication of children with autism spectrum disorder: a randomized controlled trial]. Universidade Federal do Rio Grande do Sul, Rio Grande do Sul, Brazil.

Forgeot d'Arc, B., Delorme, R., Zalla, T., Lefebvre, A., Amsellem, F., Moukawane, S., Letellier, L., Leboyer, M., Mouren, M. C., \& Ramus, F. (2016). Gaze direction detection in autism spectrum disorder. Autism (in press). doi: 10.1177/1362361316630880

Gattino, G. S. (2012). Musicoterapia aplicada à avaliação da comunicação não verbal de crianças com transtornos do espectro autista: revisão sistemática e estudo de validação (dissertation's thesis) [Music
KAMUTHE video microanalysis system for use in Brazil 
Gustavo Schulz Gattino,

Alexandre Mauat da Silva,

Felipe Grahl

Figueiredo,

Lavinia SchülerFaccini therapy assessment on nonverbal communication for children with autism spectrum disorder: a systematic review and validation study]. Universidade Federal do Rio Grande do Sul, Rio Grande do Sul, Brazil.

Gattino, G. S., Walter, F. F., \& Schüler-Faccini, L. (2010). Fundamentos sobre validade para o campo musicoterapêutico [Essentials of validity for the music therapy field]. In ASBAMT (ed.), Proceedings of the $X$ Encontro Nacional de Pesquisa em $\mathrm{Mu}$ sicoterapia [Proceedings of the X National Seminar of Research in Music Therapy] (pp. 182-190). Salvador, Bahia, Brazil: União das Associações Brasileiras de Misicoterapia.

Geretsegger, M., Elefant, C., Mössler, K., \& Gold, C. (2014). Music therapy for people with autism spectrum disorder. Cochrane Database Syst Rev, 17, CD004381. doi: 10.1002/14651858.CD004381.pub3

Grossi, D., Marcone, R., Cinquegrana, T., \& Gallucci, M. (2012). On the differential nature of induced and incidental echolalia in autism. Journal of Intellectual Disability Research, 57, 903-912. doi: 10.1111/j.1365-2788.2012.01579.x

Guillemin, F., Bombardier, C., \& Beaton, D. (1993). Cross-cultural adaptation of health-related quality of life measures: literature review and proposed guidelines. Journal of Clinical Epidemiology, 46, 1417-1432.

Kasari, C., Gulsrud, A., Freeman, S., Paparella, T., \& Hellemann, G. (2012). Longitudinal follow-up of children with autism receiving targeted interventions on joint attention and play. Journal of the American Academy of Child and Adolescent Psychiatry, 51, 487-495.

Kim, J., Wigram, T., \& Gold, C. (2008). The effects of improvisational music therapy on joint attention behaviors in autistic children: a randomized controlled study. Journal of Autism and Developmental Disorders, 38, 1758-1766. doi: 10.1007/s10803-0080566-6

Kim, J., Wigram, T., \& Gold, C. (2009). Emotional, motivational and interpersonal responsiveness of children with autism in improvisational music therapy. Autism, 13, 389-409. doi: 10.1177/ 1362361309105660

Koelsch, S. (2009). A neuroscientific perspective on music therapy. Annals of the New York Academy of Sciences, 1169, 374-384. doi: 10.1111/j.17496632.2009.04592.x

Kujala, T., Lepistö, T., \& Näätänen, R. (2013). The neural basis of aberrant speech and audition in autism spectrum disorders. Neuroscience \& Biobehavioral Reviews, 37, 697-704.

Ludlow, A., Mohr, B., Whitmore, A., Garagnani, M. Pulvermüller, F., \& Gutierrez, R. (2014). Auditory processing and sensory behaviours in children with autism spectrum disorders as revealed by mismatch negativity. Brain and Cognition, 86, 55-63.
Malerbi, F. (2008). Validação do children communication checklist (CCC) e aplicação a crianças com transtorno de déficit de atenção/hiperatividade [Validation of the Children Communication Checklist and application in children with attention deficit hyperactivity disorder] (master's thesis). Universidade de São Paulo, São Paulo, Brazil.

Mauat, A., Gattino, G., \& Riesgo, R. (2014). Validity evidences of the Individualized Music Therapy Assessment Profile (IMTAP) for ASD children (unpublished dissertation's thesis). Universidade Federal do Rio Grande do Sul, Rio Grande do Sul, Brazil.

Molnar-Szakacs, I., \& Heaton, P. (2012). Music: a unique window into the world of autism. Annals of the New York Academy of Sciences, 1252, 318-324.

Mundy, P., Kim, K., McIntyre, N., Lerro, L., \& Jarrold, W. (2016). Brief Report: Joint Attention and Information Processing in Children with Higher Functioning Autism Spectrum Disorders. Journal of Autism and Developmental Disorders, 46, 25552560. doi: 10.1007/s10803-016-2785-6

Murza, K. A., Schwartz, J. B., Hahs-Vaughn, D. L., \& Nye, C. (2016). Joint attention interventions for children with autism spectrum disorder: a systematic review and meta-analysis. International Journal of Language \& Communication Disorders, 51, 236-251.

Oldfield, A. (2006). Interactive Music Therapy in Child and Family Psychiatry: Clinical Practice, Research and Teaching. London: Jessica Kingsley Publishers.

Pell, P. J., Mareschal, I., Calder, A. J., von dem Hagen, E. A., Clifford, C. W., Baron-Cohen, S., \& Ewbank, M. P. (2016). Intact priors for gaze direction in adults with high-functioning autism spectrum conditions. Molecular Autism, 7, 25.

Pereira, A., Riesgo, R. S., \& Wagner, M. B. (2008). Childhood autism: translation and validation of the Childhood Autism Rating Scale for use in Brazil. Jornal de Pediatria, 84, 487-494. doi: 10.2223/ JPED. 1828

Plahl, C. (2004). Transactional theory on an empirical ground. Dimensions of Relation in music therapy. Music Therapy Today, $V$.

Plahl, C. (2007). Microanalysis of preverbal communication in music therapy. In T. Wosch \& $\mathrm{T}$. Wigram (eds.), Microanalysis in music therapy: methods, techniques and applications for clinicians, researchers, educators and students (pp. 41-53). London: Jessica Kinsley Publishers.

Prelock, P. J., \& Nelson, N. W. (2012). Language and communication in autism: an integrated view. Pediatric Clinics of North America, 59, 129-145.

Samson, F., Hyde, K. L., Bertone, A., Soulières, I., Mendrek, A., Ahad, P., Mottron, L., \& Zeffiro, T. A. (2011). Atypical processing of auditory temporal complexity in autistics. Neuropsychologia, 49, 546-555. 
Sperber, A. D. (2004). Translation and validation of study instruments for cross-cultural research. Gastroenterology, 126, 124-128.

Stewart, C. R., Sanchez, S. S., Grenesko, E. L., Brown, C. M., Chen, C. P., Keehn, B., Velasquez, F., Lincoln, A. J., \& Müller, R.-A. (2016). Sensory symptoms and processing of nonverbal auditory and visual stimuli in children with autism spectrum disorder. Journal of Autism and Developmental Disorders, 46, 1590-1601.

Thorup, E., Nyström, P., Gredebäck, G., Bölte, S., \& Falck-Ytter, T. (2016). Altered gaze following during live interaction in infants at risk for autism: an eye tracking study. Molecular Autism, 7, 12.

Towle, P., Farran, D., \& Confort, M. (1998). ParentHandicapped Child Interaction Observational Coding Systems: A Review. In K. Marfo (ed.), Parent-Child Interaction and Developmental Disabilities. Theory, Research, and Intervention (pp. 293366). New York: Praeger.

Travers, B. G., Adluru, N., Ennis, C., Tromp do, P. M., Destiche, D., Doran, S., Bigler, E. D., Lange, N., Lainhart, J. E., \& Alexander, A. L. (2012). Diffusion tensor imaging in autism spectrum disorder: a review. Autism Research, 5, 289-313. doi: 10.1002/ aur. 1243

Wan, C. Y., Demaine, K., Zipse, L., Norton, A., \& Schlaug, G. (2010). From music making to speaking: engaging the mirror neuron system in autism. Brain Research Bulletin, 82, 161-168.

Wigram, T. (2000). A method of music therapy assessment for the diagnosis of autism and communication disorders in children. Music Therapy Perspectives, 18, 13-22.

Wigram, T. (2002). Indications in music therapy: evidence from assessment that can identify the expectations of music therapy as a treatment for autistic spectrum disorder (ASD): meeting the challenge of evidence based practice. British Journal of Music Therapy, 16, 11-28.

Wigram, T. (2010). The religion of evidence-based practice: Helpful or harmful to health and well-being? Paper presented at the $\mathrm{V}$ European Music Therapy Congress, Cadiz, Spain.

Wigram, T., \& Gold, C. (2006). Music therapy in the assessment and treatment of autistic spectrum disorder: clinical application and research evidence. Child: Care, Health and Development, 32, 535-542.

Wigram, T., \& Lawrence, M. (2005). Music therapy as a tool for assessing hand use and communicativeness in children with Rett Syndrome. Brain \& Development, 27 Suppl 1, 95-96. doi: 10.1016/j. braindev.2005.03.019

Wigram, T., Pedersen, I., \& Bonde, L. (2002). A comprehensive guide to music therapy. London: Jessica Kinsley Publishers.

Wild, D., Grove, A., Martin, M., Eremenco, S., McElroy, S., Verjee-Lorenz, A., \& Erikson, P. (2005).
Principles of good practice for the translation and cultural adaptation process for patient-reported outcomes (PRO) measures: report of the ISPOR Task Force for Translation and Cultural Adaptation. Value in Health, 8, 94-104.

Wosch, T., \& Wigram, T. (2007). Microanalysis in music therapy: methods, techniques and applications for clinicians, researchers, educators and students. London: Jessica Kingsley Publishers.

Woynaroski, T., Watson, L., Gardner, E., Newsom, C. R., Keceli-Kaysili, B., \& Yoder, P. J. (2016). Early Predictors of Growth in Diversity of Key Consonants Used in Communication in Initially Preverbal Children with Autism Spectrum Disorder. Journal of Autism and Developmental Disorders, 46, 10131024.
KAMUTHE video microanalysis system for use in Brazil 
Appendix

\section{KAMUTHE INTERVENTION PROTOCOL MANUAL}

Objective: To assess preverbal communication abilities of children with autism spectrum disorders (ASD).

Application: Application of a thirty-minute music therapy session to conduct pre-determined activities in order to assess different forms of preverbal communication in ASD children. The session needs to be recorded (with the camera placed at a given fixed location of the room) for further analysis. The camera needs to be located in a place where the music therapist can have an overview of what happens in the setting.

Guidelines for implementation of KAMUTHE

- A trained music therapist or a music therapy student with good clinical practice skills (fully able to organize activities, sing and use percussion instruments, besides interacting with the child) is required to apply this protocol. The person responsible for applying the assessment should follow the pre-activities included in the protocol. There is a time suggestion to apply each activity, but is not necessary to follow exactly the proposed time.
- It is important that the music therapist can use strategies to approach and interact with the child such as smile, praise the child and seek physical contact (through greetings with hands, hugs and touching the shoulders, for example).

- An informal conversation with the child and the guardian will be held - in the anteroom - to receive the child, providing a safer passage from the anteroom to the setting.

- All activities seek initially spontaneous engagement of the child, starting then a verbal call and finally offering a model proposed by the music therapist (supported or not by a verbal call). From there the music therapist will remain offering the model, supported by verbal summons, until the previously established period runs out.

- In activities that involve a number of resources, they will be brought to the setting in a box and organized (with the help of the child). After the activity resources will be collected (stored), also with the help of the child.

\section{ACTIVITIES}

Initial conversation (about $1 \mathrm{~min}$ ): the conversation starts with "hello", "good morning" or "good afternoon”. Possible questions: "How are you?", "What is your name?", "Do you like music?", "Which song/ singer do you like?", "Which team do you support?", "Who came with you today?", "How did you come here?", "Do you have brothers?", "How was school?", "What do you want to do today?" (Musical preferences, preferred instruments, activities, etc.).

Hello song (about 2 min): This song could be an entrance or welcome song. This song marks the beginning of the session and should aim to facilitate vocalizations, speech, singing, smiles and gestures. This process can be facilitated through a simple melodic and harmonic structure (repeating itself), as well as a text of a few sentences (maximum three). Expressions such as "hello", "good morning", "good afternoon" in addition to the child's name should be part of the text of the song. Still under the harmony of the entrance song (after greeting the child), the music therapist can ask questions like, "What would you like to do today?", "Singing?", "Dancing?", "Playing the keyboard?" (start with open-ended questions and, if necessary, facilitate interaction by answering "yes/no").

Sounding the interests of the child (about $10 \mathrm{~min}$ ): This activity aims to locate the child's interests, considering that desire is an important fac- tor for participation. The activity can be based on composition techniques or musical improvisation. The goal is to engage with the child with vocal and gestural participation. 1) the first part of this activity is following the child's interests using the voice and the guitar according to the child's productions (vocal, body and sounds produced by objects - in a table, chair, etc). If the child responds well to the musical proposal from the music therapist, it is necessary to deepen the musical play, creating a musical space for new possibilities of interaction to engage different preverbal communication. While the music therapist plays, he may invite the participant to play.

Playing with objects (about 6 min): This activity aims to explore use of a nonmusical object to produce music in a musical improvisation experience. During the activity, the music therapist needs to follow the child's lead, performing a support in the musical play. This support can be given through a musical instrument, vocalizations, singing a wellknown song of the participant and/or by instrumental improvisation. The music therapist may also include the name of the child in care in songs. The activity will consist of the following: 1) to seek the object along with the child; 2) Expect the initiative of the child to touch the object; 3 ) If the child does not, the music therapist gives a verbal request (twice) for the child to touch the object as a tool; 4) If the child 
does not touch the object, the music therapist offers a "model of how to play" for the child and gives the child a suggestion to make the touch. A suggestion of an object could be a plastic bucket.

Activity with musical instruments (about $10 \mathrm{~min}$ ): This activity aims to connect the child with different musical instruments. This activity creates opportunities for the child to choose, explore and touch distinct musical instruments. During the activity, the music therapist needs to follow the child's lead, performing a support in the musical play. This support can be given by playing musical instruments, vocalizations, singing a well-known song, and music instrumental improvisation. The music therapist may also include the name of the child in care in songs. The activity will consist of the following: 1) search and organize musical instruments along with the child. The instruments are guitar, keyboard and drum; 2) wait for the child to make their choice freely; 3) call (verbally) the child to make a choice, suggesting that she take, manipulate, touch the instruments; 4) in the absence of answers, the music therapist chooses one of the instruments, offering a model of how to play and making some musical suggestions.

Goodbye song (about $1 \mathrm{~min}$ ): The goodbye song marks the end of the session and should aim at facilitating vocalizations, speech, singing, smiles and gestures. This process can be facilitated through a simple melodic and harmonic structure (repeating itself), as well as a text of a few sentences (maximum two). Expressions such as "bye", "goodbye", as well as the child's name should be part of the text of the farewell song.

The activities described above will allow the observation of all categories and specific behaviors mentioned in the KAMUTHE system: child categories and music therapist categories. 